\title{
Role of nasal casts for in vitro evaluation of nasal drug delivery and quantitative evaluation of various nasal casts
}

\author{
Per G Djupesland*,1 (iD), John C Messina2 (iD) \& Ramy A Mahmoud² (iD \\ ${ }^{1}$ OptiNose AS, Oslo, Norway \\ ${ }^{2}$ OptiNose US, Inc., Yardley, PA 19067, USA \\ *Author for correspondence: Tel.: +47 48090 610; per.djupesland@optinose.com
}

Background: Nasal casts may characterize intranasal drug deposition. Methodology: The Koken cast, described as 'anatomically correct', and the Optinose cast, derived from MRI of a healthy male during velum closure, were dimensionally compared and assessed for deposition assessment suitability. Results: Smallest vertical cross-sectional areas (valve region) for Koken and Optinose right/left: $2.55 / 2.75$ and $1.18 / 1.18 \mathrm{~cm}^{2}$, respectively, versus a 'normative' mean (range) of $0.85 \mathrm{~cm}^{2}\left(0.2-1.6 \mathrm{~cm}^{2}\right)$. Intranasal volumes differed (computed tomography/water fill): Koken, $35.8 / 38.6 \mathrm{~cm}^{3}$ and Optinose, $24.1 / 25.0 \mathrm{~cm}^{3}$, versus a 'normative' mean (range) of $26.4 \mathrm{~cm}^{3}\left(20.9-31.1 \mathrm{~cm}^{3}\right)$. Conclusion: Koken cast dimensions are larger than the normal range and the Optinose cast. The validity of casts for regulatory drug deposition studies is suspect.

First draft submitted: 6 May 2020; Accepted for publication: 13 July 2020; Published online:

30 July 2020

Keywords: devices • in vitro • nasal • targeting organ

The pattern of intranasal drug deposition produced by different delivery mechanisms can have a material impact on the consequences of drug treatment: different delivery mechanisms have been shown to result in different pharmacokinetic profiles for multiple drugs, and delivery differences may be even more important for topically acting medications [1-3]. Differences in drug delivery are well understood to have an important influence on efficacy and safety of medications inhaled into the lower respiratory tract. Drug delivery is comparably important for comparative assessment of drugs administered to the upper respiratory tract. Therefore, assessment of differences in nasal drug deposition achieved by different delivery approaches is important in developing innovative approaches to intranasal drug delivery, particularly with topically acting medications, as well as for comparison with prior approaches. Although in vivo evaluation in humans is self-evidently preferred, the low cost and speed of in vitro evaluation with nasal cast models makes them tempting for evaluation of nasal delivery performance [4].

Achieving high and deep intranasal drug deposition is difficult even without nasal pathology. The anatomy of the nasal labyrinth is complex, with multiple convoluted and obstructing surfaces, meatuses, ostia and slit-like passages. Furthermore, the anatomy is dynamic, with changing dimensions during normal inspiration/expiration and with the nasal cycle [5-7]. These factors not only make delivery difficult, but also complicate accurate in vitro simulation of in vivo performance [8].

Current regulatory guidance recommends that several in vitro tests (e.g., emitted dose, droplet or particle size distribution, spray pattern, plume geometry) may be used to characterize or compare nasal drug products in support of applications for new drugs (new drug applications) or for generic drugs [6,9]. These recommended in vitro tests assess the quantity of drug delivered and the reproducibility of certain characteristics of drug delivered by a spray device; for example, attributes of a typical expanding aerosol spray plume (e.g., plume geometry, spray pattern), even though such in vitro expanding plumes clearly cannot develop in vivo inside the narrow nose $[6,8]$.

These in vitro methods are useful for informing issues related to reliability of manufacturing or for certain kinds of discrimination among products [6]. However, due to the static and dynamic complexity of the nasal labyrinth and the dynamics of the narrow, but flexible, nasal valve, it is not at all obvious that these, or other currently available, in vitro tests can serve as accurate predictors of human in vivo deposition, absorption, or clinical response [8]. 

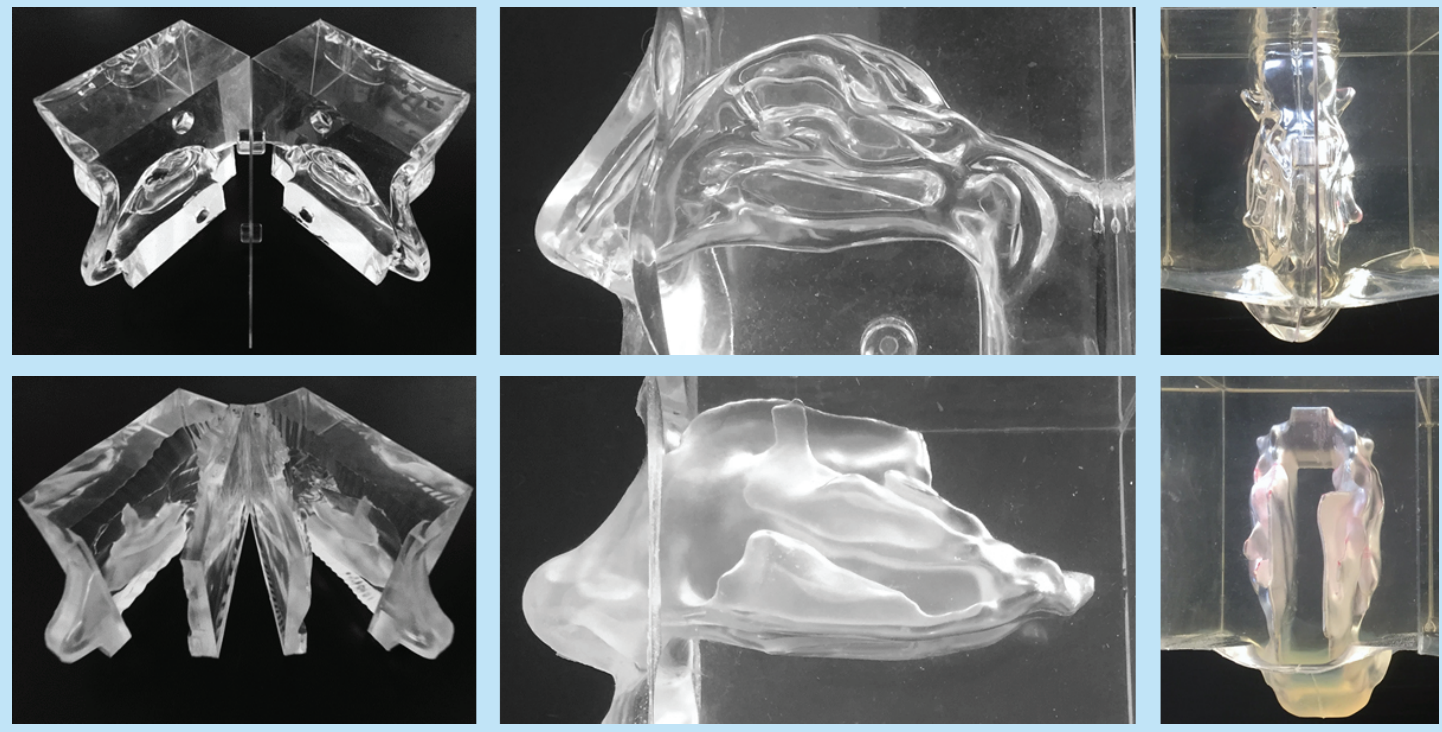

Figure 1. Casts. (Top) Koken cast. (Bottom) Optinose cast. Far left panels show the casts opened. The Koken cast is split in two silicone parts and a central, thin, flat, transparent, plastic septum separating the nasal cavities. The Optinose cast is split in four parts: two lateral parts and two central parts constituting the boarded septum with the true geometry of the medial sides of the nasal septum. Middle panels show lateral view of the two silicone casts. Far right panels show superior view of the two casts.

Because of the attraction of using in vitro simulation of drug delivery instead of more definitive in vivo testing, methods for standardization of cast properties and testing procedures have been proposed as a means of using in vitro testing in nasal casts for regulatory assessment of bioequivalence of delivery between products $[4,9]$. Unfortunately, current nasal cast models - though helpful approximations for early product development - may be fundamentally inadequate for accurate simulation of the human nasal cavity in vivo. This is because studies of deposition profiles using nasal casts are at risk of systematically mischaracterizing human in vivo performance. In particular, cast studies are likely to underestimate the difficulty of delivering nasally administered drugs beyond the nasal valve. Delivery to posterior/superior surfaces may be overestimated and types of epithelium receiving drug mischaracterized $[4,8,10]$.

The only commercially available human nasal cast (Koken Co., Tokyo, Japan) is, according to the manufacturer, primarily an educational tool. It is based on a cadaver and has a flat, transparent septum to enable the visualization of nasal structures (Figure 1 [top]). On inspection, both the dimensions and shape of the nasal valve area observably deviate from normal patient anatomy [11].

An alternative silicone cast has been developed, intended to more accurately reproduce nasal geometry (Figure 1 [bottom]). The Optinose cast (OptiNose AS, Oslo, Norway) features a closed velum (soft palate), allowing for testing of both conventional nasal drug delivery and the more recently developed exhalation delivery system (EDS), through which delivery of drug occurs with a closed velum (Figure 2) [12]. This cast is currently not commercially available.

In this study, we compared the dimensions and geometry of these two casts with each other, using imaging, 3D modeling and water fill, and we assessed the validity of the casts for comparison to in vivo conditions. We also compared the two casts to normative values from published literature and reviewed some limitations of cast models for accurate simulation of nasal drug delivery.

\section{Methodology}

Koken nasal model (cast)

The Koken silicone cast (Koken Co., www.kokenmpc.co.jp/english/products/life_simulation_models/medical_edu cation/lm-005/) is based on an Asian female cadaver. It can be split in two halves and has a flat, transparent, removable septum to enable visualization of internal nasal structures (Koken Co.) (Figure 1 [top]). Because it is commercially available and readily accessible, it has been used for deposition studies in multiple peer-reviewed 


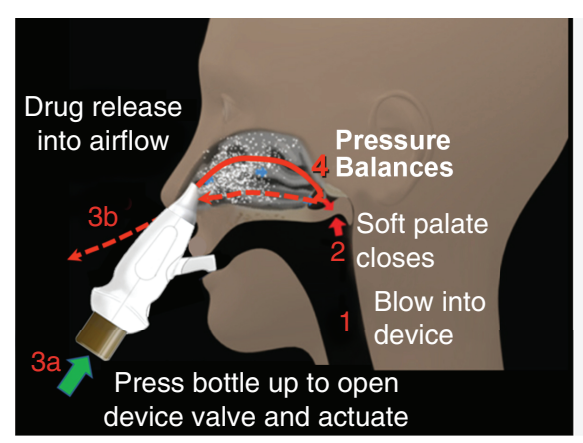

Sagittal plane

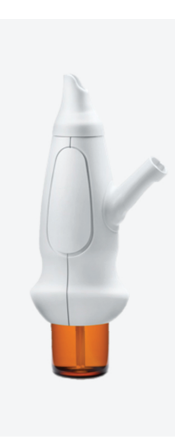

Transverse plane

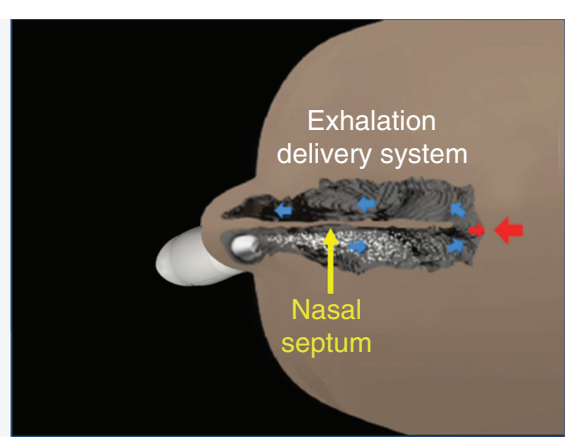

Figure 2. Mechanism of exhalation delivery system. Reproduced with permission from [12].

Figure 3. Nasal valve secretion and collapse in MRIs used to develop the Optinose cast. Cross-sections in the nasal valve region from MRI used to develop Optinose cast. The images show secretion partly obstructing the upper slender part of the valve and partial collapse of the upper part of the valve region on the left side.

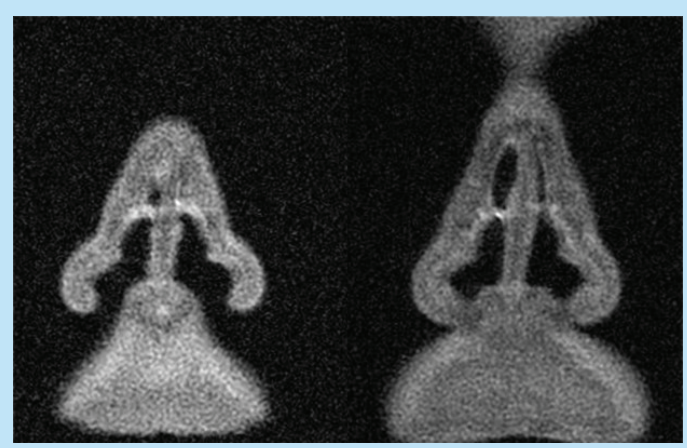

publications [4,12-17]. This cast has been described in some publications as 'anatomically correct' [13-17]. However, no validation of the geometry and dimensions of this cast have been presented in publications to date.

\section{Optinose nasal model (cast)}

An alternative cast (Optinose AS) (Figure 1 [bottom]), intended to more accurately reproduce human intranasal geometry and dimensions, was developed using 3D computer reconstruction and surface rendering from highresolution MRI in a nondecongested, healthy, 26-year-old male. During imaging, the soft palate was elevated by exhaling through the mouth against resistance. This action maintains an airtight seal of the velum separating the oral and nasal cavities and simulates the oropharyngeal anatomy during use of an EDS (Optinose AS) (Figure 2) [12,18].

For the purposes of this cast, using a computer-aided design program, the two nasal cavities were parallel shifted by $8 \mathrm{~mm}$ to allow the septum to be split into two parts with sufficient thickness/rigidity to maintain the septum's internal geometry while not changing the internal geometry of the nasal cavities on either side. The communication between the two sides of the nose (behind the posterior margin of the nasal septum) was therefore also extended by $8 \mathrm{~mm}$ but still allowed for communication between the nasal cavities during drug delivery. As shown on the MRI section in Figure 3, the upper narrow section of the nasal valve was naturally partly collapsed and occluded by normal secretions at the time of imaging, as is frequently true during rest and inspiration. To maintain a fluid and dynamic pathway that would allow realistic valve behavior (e.g., expansion during airflow and drug delivery, stenting by nosepiece), the two walls of the valve were separated slightly in the computer-aided design model. This type of correction has also been performed on computed tomography (CT) scans in other studies [19]. This procedure increases the cross-sectional area to some extent compared with in vivo dimensions of the valve as measured by acoustic rhinometry (AR) (Table 1); however, it is representative of what actually occurs during insertion of an EDS nosepiece, during exhalation delivery and during nasal exhalation [20,21]. A detailed replica of the complex nasal geometry was then made with a stereolithographic 3D printer (Objet 250, CATI, IL, USA). This geometry was 


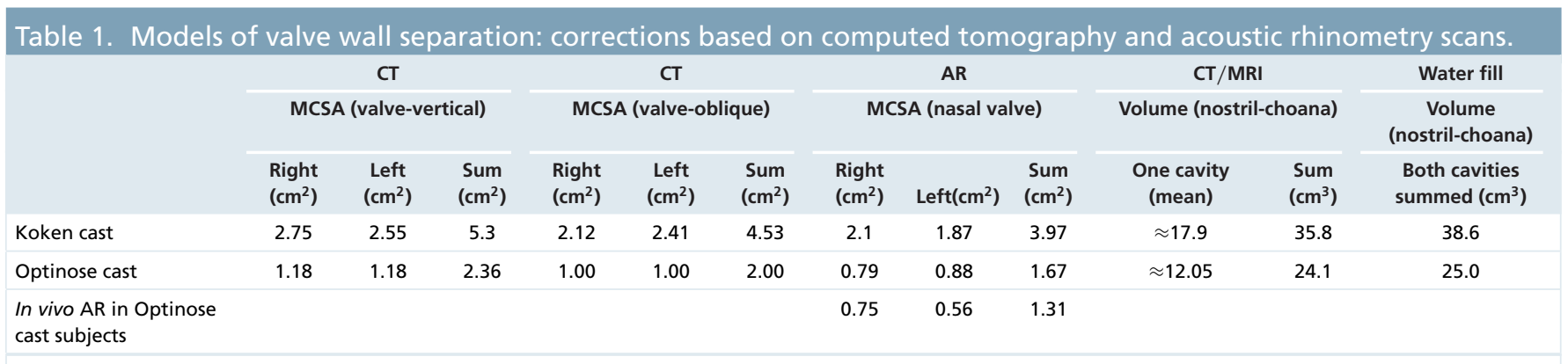

AR: Acoustic rhinometry; CT: Computed tomography; MCSA: Minimum cross-sectional area.

placed in a box subsequently filled with fluid, semi-transparent, semi-soft silicone (Andersen, Jessheim, Norway). After drying, the cast was carefully broken into pieces and removed. The semi-transparent silicone material allows visualization of internal structures from different angles. In a variant, a cast was sectioned into four parts that can be separated, allowing concurrent direct visualization of the geometry and deposition in the medial and lateral aspects of both sides of the nose from 'inside the nose'. When the parts were reassembled, the nasal cavities inside the silicone block mirrored the original nasal airway (Figure 1 [bottom]).

\section{Assessment of the geometry of both nasal models}

The Koken and Optinose silicone casts were placed on the examination table of a 64-slice GE LightSpeed VCT scanner (GE Healthcare, IL, USA) and oriented such that axial slices would show the nasal cavity in sections vertical to the nasal floor and with the nasal septum in a sagittal plane. A 'paranasal sinus' protocol with 'bone' algorithm with a $0.625-\mathrm{mm}$ slice thickness in three planes was performed $(120 \mathrm{kV}, 700 \mathrm{~mA}, 1460$-ms rotation time, 0.531 pitch, with CT dose index of $80.74 \mathrm{mGy}$ ) and the data saved as DICOM files.

\section{Building 3D models of nasal cavities}

The open-source medical imaging software, Slicer (www.slicer.org), was used to create 3D surface-model DICOM files of the nasal cavities of both casts. The DICOM files were loaded, and, using the image editor, the nasal cavities of each image were colored. Using the Slicer module, 'Model Maker', a 3D surface model was generated and exported to a stereolithography file. The stereolithography surface models, made up of thousands of small triangles, were imported into 3D software (3D-Tool GmbH \& Co., Weinheim, Germany), transformed into sections based on position and angle and exported as $2 \mathrm{D}$ drawing exchange format files. The drawing exchange format files define the outline of the sections as multiple, connected line segments. 3D solid-modelling software (SolidWorks, MA, USA) was then used to create and calculate the cross-sectional areas of the left and right nasal cavity perpendicular to the nasal floor at 1 -mm intervals in both casts. In addition to the perpendicular cross-sectional areas in the nasal valve region, the smallest oblique cross-sectional area $\left(\sim 30^{\circ}\right.$ tilted forward $)$ in this region was estimated to allow a more realistic comparison with measurements by AR (see next section) [22].

\section{Acoustic rhinometry}

$\mathrm{AR}$ is a noninvasive technique for determining cross-sections in the nose by analyzing changes in emitted and reflected sound impulses [23]. Cross-sections are measured along a curved acoustic pathway and are therefore not directly comparable to CT/MRI-inferred cross-sections vertical to the nasal floor, notably in the nasal valve region [22,23]. The minimum (narrowest) cross-sectional area (MCSA; mean of three independent measurements) in the anterior nasal valve region (approximately between 1.5 and $3.5 \mathrm{~cm}$ from the nostril) was assessed by AR (SRE21000, RhinoMetrics A/S, Lynge, Denmark) in both the subject from whom the Optinose cast was generated and in both casts. The MCSA on each side of the nose varies constantly with the nasal cycle, but the bilateral summed area remains fairly constant [7]. Volumes were not estimated by AR because reliability is reduced behind narrow constrictions and beyond $5 \mathrm{~cm}$ from the nostril due to potential sound loss to sinuses [22,23].

\section{Cast volume determination}

The nostrils of the casts were sealed with soft silicone, and the casts were positioned with the nose pointed downward and the floor of the nose perpendicular to the horizontal plane. A filling needle was inserted in the slits between the 
sections of the casts, and colored water was slowly injected into the nostrils until reaching the level of the posterior margin of the septum. The total volume of the combined (bilateral) nasal cavities was determined by the amount of liquid required to fill each cast from the nostril to the level of the posterior margin of the septum (the choana).

\section{Results}

The dimensions of the two different nasal models, as assessed by CT scanning and AR, were not similar: crosssectional dimensions and volumes of the Koken cast were substantially larger than the Optinose cast (Table 1 $\&$ Figures $4 \& 5$ ). The unilateral and summed cross-sectional areas, measured vertically to the nasal floor and horizontally at the smallest oblique cross-sectional area in the valve region, for the two casts are shown in Figure 4 and by curves for the summed MCSAs along the curved acoustic pathway measured by AR in Figure 5 .

The distance from the anterior margin of the nostril to the posterior margin of the septum (choana) was approximately 7 and $8 \mathrm{~cm}$ for the Koken and Optinose casts, respectively (Figure 4). The intranasal volumes (nostril to the choana) of the two casts were markedly different as assessed by both CT and water fill: the Koken model had a volume of $35.8 / 38.6 \mathrm{~cm}^{3}$ (CT/water fill), whereas the Optinose model had a volume of $24.1 / 25.0 \mathrm{~cm}^{3}$ (Table 1 \& Figure 5).

In addition to abnormally large cross-sectional dimensions and volumes, the nasal valve of the Koken cast was observed to have an oval shape (Figure 4). This is not consistent with the slender triangular shape of the normal in vivo nasal valve (Figures $3 \& 4$ ).

\section{Discussion}

The potential of any nasal cast model in helping predict the in vivo performance of an intranasal delivery method is highly dependent on how representative the model is of in vivo nasal anatomy, as well as how closely the dynamic characteristics of the nose are replicated in the cast. This study compared the quantitative and qualitative attributes of two nasal cast models and showed that the Koken cast has intranasal dimensions that are substantially larger than the Optinose cast, as well as displaying larger-than-normative data for both nasal volume and MCSA. Unlike the Koken model, the nasal volumes, cross-sectional dimensions and nasal valve shape of the Optinose model were more representative of the normal range for both genders described by the Carleton-Civic Standardization curve and the supportive normative data derived from multiple published studies (Figure 6) [19].

\section{Nasal volumes}

The volume of the nasal cavity in vivo has been previously reported. In one study of healthy individuals, the mean internal volume of standard nasal geometry was reported to be a mean of $13.19 \mathrm{~cm}^{3}$ (standard deviation \pm 2 ; range, 10.1-16.3) or a summed bilateral volume of $26.4 \mathrm{~cm}^{3}$ (range 20.9 to $31.1 \mathrm{~cm}^{3}$ ) in the seven subjects with available volume data) [19]. The validity of these volumes is supported by a separate report in which MRI-derived volumes of 45 human subjects ( 22 males and 23 females) were found to be similar (mean $8.9 \mathrm{~cm}^{3}$; range 7.37 to $16.91 \mathrm{~cm}^{3}$ ) [24]. In the latter report, there were no significant differences between sides and no gender-related differences in intranasal volume that were not accounted for by subject size (height and head size) [24].

Comparisons of these data with the casts used in this study show that the internal volume associated with the Koken cast is far outside (above) the normal range, whereas the values for the Optinose cast were well within the normal range (Table 1 \& Figure 6) [19].

\section{Minimal cross-sectional areas}

As measured by CT, the smallest unilateral cross-sectional area vertical to the nasal floor as measured in the Optinose cast (right/left) was $1.18 / 1.18 \mathrm{~cm}^{2}$; when tilted, the smallest cross-sectional area in the valve region was reduced to $1.0 / 1.0 \mathrm{~cm}^{2}$ (Table $1 \&$ Figure 4). These values are well within the 'normative' range for men and women (unilateral mean $\sim 0.85 \mathrm{~cm}^{2}$; range $\sim 0.2$ to $1.6 \mathrm{~cm}^{2}$ inferred from CT scan) [19]. As assessed by AR, the MCSAs of the Optinose cast were also well within the normal range of unilateral MCSA $\left(0.4-1.17 \mathrm{~cm}^{2}\right)$, as reported in a review of multiple AR studies in a total of more than 1000 healthy adults of both genders (mean unilateral MCSA: $0.52-0.78 \mathrm{~cm}^{2}$; see Table $1 \&$ Figures $4 \& 5$ ) [23,25]. In a recent CT study in 22 subjects, the mean summed MCSA measured by AR (ages 21-39; male:female $=10: 12$ ) was $2.0 \mathrm{~cm}^{2}$, which was very similar to the AR dimensions of the casts and the oblique smallest cross-sectional area in the Optinose cast (Table 1 \& Figures 4 \& 5) [26]. Interestingly, computational fluid dynamics simulations of multiple nasal airflow features in these CT geometries 


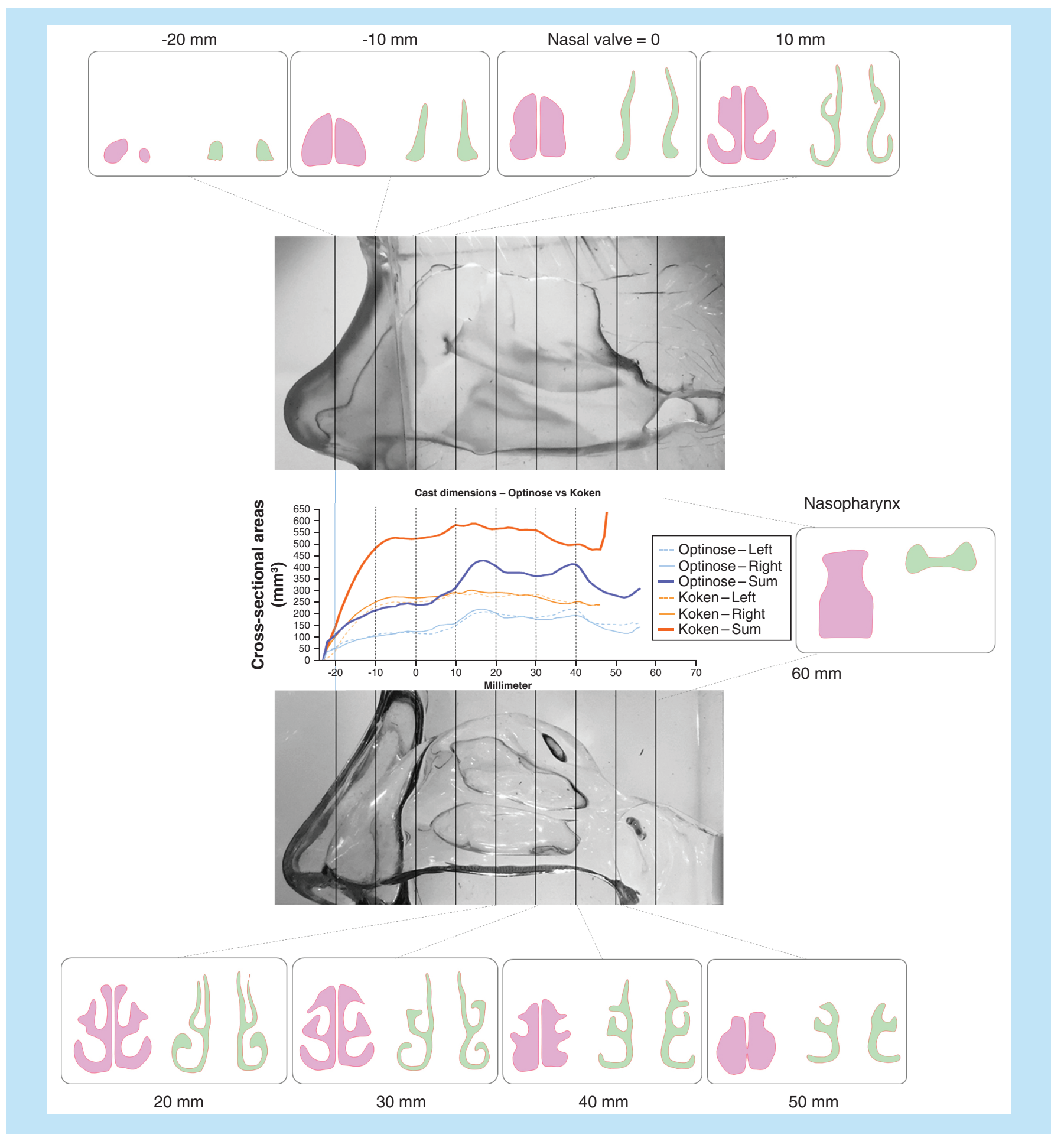

Figure 4. Comparison of shape and dimensions of cross-sections in the two casts. The location of the smallest cross-sectional area in both casts is set to 0 on the $\mathrm{x}$ axis to allow for the most realistic comparison of dimensions. The smallest oblique cross-sectional area was at an angle of approximately $20^{\circ}$ to the nasal floor. 


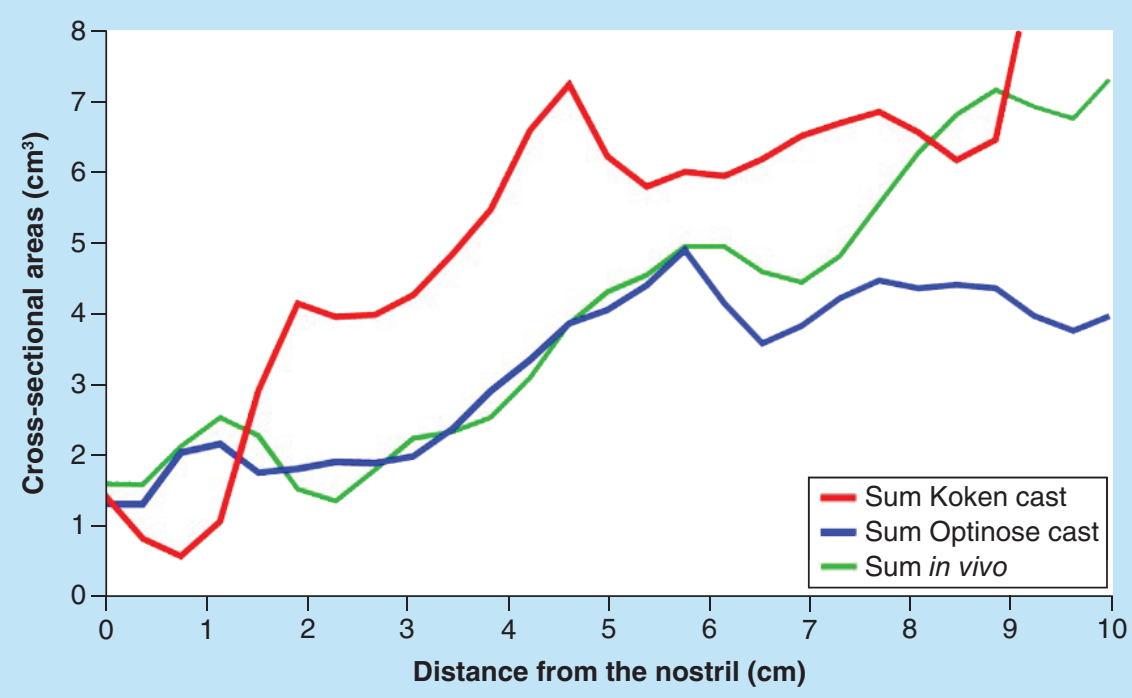

Figure 5. Acoustic rhinometry curves in vivo and in the two casts. Summed dimensions as a function of distance along the acoustic pathway in the two casts and in vivo in the subject from whom the Optinose cast was generated as measured by acoustic rhinometry.
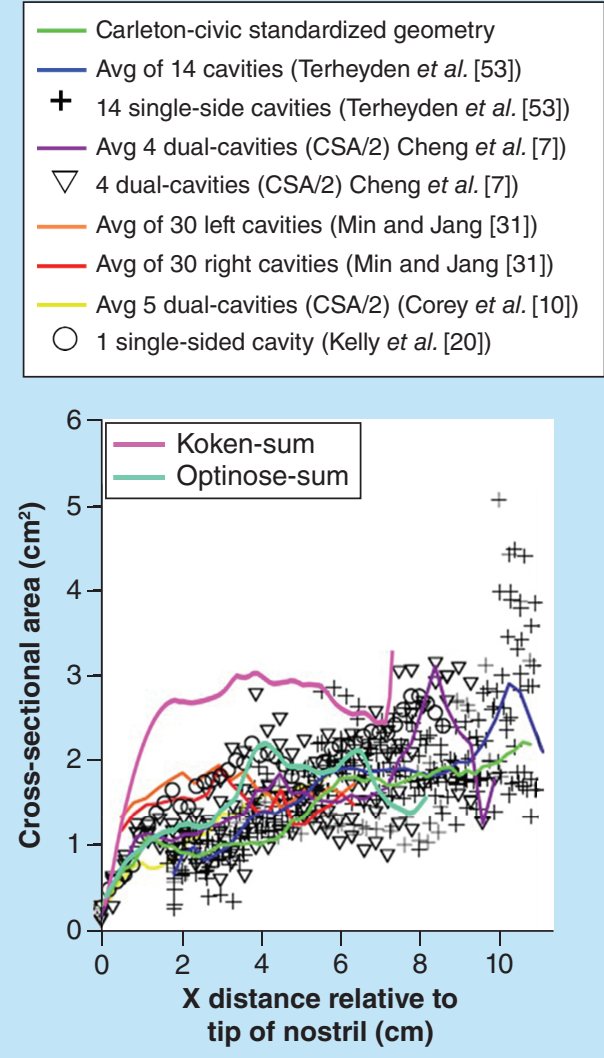

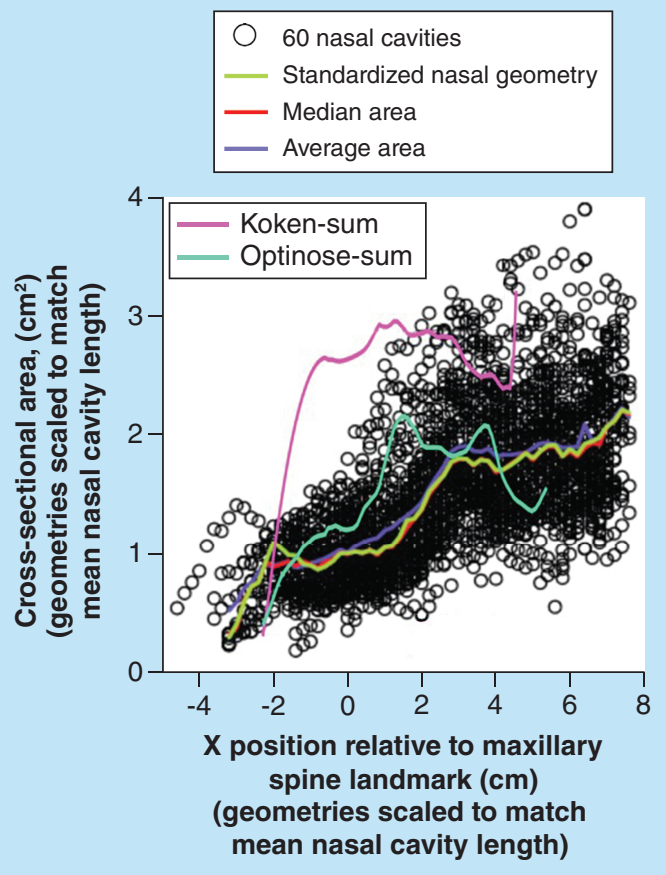

Figure 6. Koken and Optinose cast dimensions measured in computed tomography scans (mean of right and left cavity) superimposed on graphs from Liu et al. (Left) Unilateral nasal dimensions from multiple published studies collated. (Right) Unilateral nasal dimensions from 60 nasal cavities.

Adapted with permission from [19]. 
were performed, and the authors verified the key role of the nasal valve in defining nasal aerodynamics and function, concluding that a 'too-wide' nasal valve may negatively alter nasal aerodynamics [26].

The nasal valve area is an important area in the context of assessing deposition of intranasally administered medication, because it typically prevents particles and droplets from reaching the superior regions of the nasal cavity. Despite the wide range of 'normal' nasal dimensions, the Koken model still has artefactual, excessively high cross-sectional areas: valve dimensions are twice the Optinose cast and far outside the normal range (Table 1 \& Figures 4-6) [19,25]. This is likely due to post-mortem changes and, because the Koken cast was designed for educational use, potentially due to modifications to the valve and other geometry during manufacturing to enhance visual access. In contrast, the Optinose model's dimensions are within the range of normal and are therefore more likely to accurately represent static in vivo anatomy.

The shape of the nasal valve region is as important as the size. The valve region of the Koken cast is oval shaped and deviates substantially from a normal in vivo anatomic configuration. This difference would be expected to strongly influence nasal flow and pressure characteristics, as well as the deposition patterns of nasal delivery devices, particularly those with an expanding plume $[20,21,26]$.

Based on these results, the validity of the Koken cast to model, inform, or compare drug delivery, including drug deposition profiles, of different nasally delivered products (as has been reported in multiple publications) must be called into question $[13,15-17]$.

\section{Issues influencing relationship to in vivo use}

If one wishes to create in vitro models to simulate human in vivo nasal conditions (e.g., to simulate deposition patterns produced by different drug delivery approaches), then the accuracy of dimensions and geometry of the nasal casts relative to normal nasal anatomy are obviously important. These data show that the Koken cast is outside normal values for nasal volume and nasal valve area, whereas the Optinose cast falls within normal values for both parameters $[13,15-17]$. However, it is important to remember that accurate dimensions and geometry alone are not sufficient to simulate in vivo behavior of the nose during drug delivery [8].

Both of these casts were made of silicone. The slippery and firm surface of silicone is quite unlike the normal tissues lining the nose in vivo and thus presents both a static and dynamic problem for in vitro assessment of deposition in a cast model. A surface coating may mitigate part of this problem but only to a limited extent. The differences in moisture and surface texture, lack of mucociliary clearance, lack of cyclical changes in nasal cavity dimensions (i.e., the normal nasal cycle) and mucosal sensitivity to temperature and to mechanical impact are additional factors that negatively influence the ability to assess nasal performance (e.g., drug deposition) in vitro with a cast model as a surrogate for accurate assessment of deposition in vivo.

Real-life in vivo results may also differ from what is simulated in casts due to human behavior variation that may also influence deposition [6,15]. For example, patients using intranasal sprays often sniff due to instructions or simply due to feeling 'drip-out', resulting in transport of medication along the nasal floor. Sniffing also affects nasal valve dynamics, potentially causing valve collapse. This, however, is not an issue with the EDS device, as positive pressure is applied, and the nasal valve is mechanically expanded. The depth of insertion and angle of devices in the nostril may also differ in real-life use compared with simulation in casts. These factors may be particularly important in situations in which device placement may sometimes produce discomfort/pain, as devices contact sensitive nasal tissues (e.g., the turbinate) - an effect that is challenging to simulate in silicone casts.

Another feature of the Koken cast geometry that may lead to misrepresentation of flow and deposition characteristics associated with intranasal delivery is the flat septum. Additionally, the nostril entrances in the Koken cast are unnaturally narrow, requiring mechanical enlargement in some studies and creating additional risk to accurate simulation of in vivo structure and function (Figure 5) [13]. To maintain the appropriate septal contours, the two sides of the nose in the Optinose cast were shifted $8 \mathrm{~mm}$ apart to allow sufficient material thickness. Although this parallel extension of the communication behind the septum could potentially change aerodynamics posterior to the septum (an 8-mm elongation, or 'tunnel', behind the septum), the impact is likely to be minimal [12].

\section{Flow dynamics}

Inhalation and exhalation through the nose normally have dynamic effects and change the volume of unoccupied space in the nasal cavity due to the soft nature of the structures resulting from both muscle activity (e.g., flare) and Bernoulli's principle. Rigid or semi-rigid nasal casts cannot replicate the dynamics of soft nasal tissues and, in particular, of the narrow valve area. With conventional nasal sprays, inhalation ('sniffing') is both a common part 
of instructions for use and a likely consequence of the user's desire to draw drug into the nose and prevent 'drip out'. In vivo, as inhalation flow rates increase through the narrow nasal valve region, a further narrowing, or even a collapse, of all or part of the region can result from the negative pressure created by Bernoulli's principle. This has been documented by flow and pressure measurements and by endoscopy $[4,6,21]$. Collapse due to Bernoulli's principle cannot be simulated with currently available silicone casts. Furthermore, when a user sniffs during or after intranasal administration of drug - creating negative pressure by drawing air into the throat and lungs - the aerosol liquid or particles are drawn primarily along the path of least resistance along the nasal floor under the inferior turbinate, passing through the less constricted -and more likely still patent - inferior portion of the nasal valve $[6,27,28]$. Similarly, with exhalation delivery, a positive pressure being introduced to the nose would actively expand the soft tissues and likely impact drug deposition characteristics. However, because of the rigidity of the silicone casts, the impact of this effect on deposition would not likely be observed. Irrespective of their 'static' anatomic correctness, the importance of the dynamic functional characteristics of the nose suggests that relatively rigid silicone cast models are not well suited to accurate, definitive assessment of in vivo deposition.

\section{Conclusion}

Based on multiple methods of assessment, it is clear that the educational Koken cast does not have normal dimensions or a normal shape in multiple key regions. In contrast, the dimensions of the Optinose cast and nasal valve shape are within the normal range of in vivo dimensions. Models with dimensions and shape within the normal range may improve in vitro simulation of human in vivo drug deposition patterns with various drug delivery mechanisms of action. Due to their transparency, these types of casts can be helpful in early evaluations and provide a relatively easily understood visual indication of performance. However, even anatomically representative casts are generally not suitable when an accurate, definitive scientific assessment of nasal deposition is required, as expected for regulatory purposes. Alternatives such as 'in silico' computerized fluid dynamic simulation or other newer developments may, in the future, with suitable validation, present complementary approaches to current modelling, although the complexity and wide degree of variability in the nasal labyrinth will likely continue to pose a significant assessment challenge [26]. For the time being, and in contrast to what has been previously proposed [4,15], there is no substitute for in vivo assessment when a definitive determination of nasal delivery performance is required (e.g., for 'bioequivalence'). This is particularly essential in the context of medications with at least some degree of topical activity, for which the pattern of deposition is expected to strongly influence safety and/or efficacy.

\section{Author contributions}

PG Djupesland contributed to study conception and design, acquisition of data, analysis and interpretation of data, drafting of manuscript and critical revisions. JC Messina and RA Mahmoud contributed to study conception and design, analysis and interpretation of data, drafting of manuscript and critical revisions.

\section{Acknowledgments}

The authors would like to thank HK Andersen, medical physicist, Intervention Centre, Oslo University Hospital. She was responsible for acquiring and providing the high resolution MRI-scan. We would also like to thank M Iversen for data analysis and 3D-model development.

Data in part presented at COSM 2017 (Combined Otolaryngology Spring Meetings, 26-30 April 2017; San Diego, CA, USA).

\section{Financial \& competing interests disclosure}

This study was funded by OptiNose. JC Messina reports being an employee and shareholder of OptiNose during the conduct of the study. RA Mahmoud reports being an employee and shareholder of OptiNose during the conduct of the study; in addition, he is a listed inventor on nasal delivery device patents. PG Djupesland reports being an employee and shareholder of OptiNose during the conduct of the study, and he was a board member of OptiNose AS during the conduct of the study; in addition, he is a listed inventor on nasal delivery device patents. OptiNose AS received grants from the Norwegian Research Council during the study and a tax deduction from SkatteFUNN related to the study. The authors have no other relevant affiliations or financial involvement with any organization or entity with a financial interest in or financial conflict with the subject matter or materials discussed in the manuscript apart from those disclosed.

Editorial assistance with drafting the report following the authors' guidance, incorporating comments according to authors' feedback and providing support with submission was provided by ECIR Medical Communications (BJ Epstein, President; LM Ritter, Scientific Director), funded by OptiNose US, Inc. 


\section{Ethical conduct of research}

The authors state that consent was provided for use of MRI. No additional relevant information regarding the ethical conduct of their research.

\section{Open access}

This work is licensed under the Attribution-NonCommercial-NoDerivatives 4.0 Unported License. To view a copy of this license, visit http://creativecommons.org/licenses/by-nc-nd/4.0/

\section{Summary points}

\section{Nasal casts for simulating drug deposition}

- Differences in drug delivery and deposition may have an important influence on efficacy and safety of medications delivered to the upper respiratory tract.

- It has been suggested that nasal casts may be useful for characterizing intranasal drug deposition during regulatory assessment.

- The only commercially available nasal cast, the Koken cast, is based on a female cadaver and has been described as 'anatomically correct'. The Optinose cast is derived from magnetic resonance imaging of a healthy male during velum closure.

Assessment of the geometry of the Koken \& the Optinose nasal casts

- The dimensions of the the Koken and the Optinose nasal models, as assessed by computed tomography scanning and acoustic rhinometry, are not similar: cross-sectional dimensions and volumes of the Koken cast are substantially larger than the Optinose cast.

- The intranasal volumes (nostril to the choana) of the two casts are also markedly different as assessed by both computed tomography and water fill.

- The nasal valve of the Koken cast has an oval shape, inconsistent with the slender triangular shape of the normal in vivo nasal valve.

In vitro simulation of human in vivo drug deposition patterns

- Unlike the Koken cast, the nasal volumes, cross-sectional dimensions and nasal valve shape of the Optinose cast are within the normal range of in vivo dimensions for both genders. Koken cast dimensions are substantially larger than the normal range.

- The validity of drug deposition data produced using the Koken cast, or any nasal cast, are questionable for predicting in vivo deposition.

- While nasal casts with dimensions and shape within the normal range may improve in vitro simulation of human in vivo drug deposition patterns with various drug delivery mechanisms, static, dynamic and behavioral factors suggest that there is no substitute for in vivo assessment when a definitive determination of nasal delivery performance is required, as expected for regulatory purposes.

\section{References}

Papers of special note have been highlighted as: $\bullet$ of interest; $\bullet \bullet$ of considerable interest

1. XHANCE, prescribing information. Optinose US; Yardley, PA, USA (2017).

2. Messina J, Carothers J, Obaidi M et al. Intranasal fluticasone propionate delivered by exhalation delivery system (FLU-EDS) versus Flonase ${ }^{\circledR}$ nasal spray and Flovent ${ }^{\circledR}$ HFA: a randomized comparison of bioavailability. J. Allergy Clin. Immunol. 139(Suppl. 2), AB253 (2017).

3. Obaidi M, Offman E, Messina J et al. Improved pharmacokinetics of sumatriptan with Breath Powered ${ }^{\mathrm{TM}}$ nasal delivery of sumatriptan powder. Headache. 53(8), 1323-1333 (2013).

4. Azimi M, Longest PW, Hindle M. Towards clinically relevant in vitro testing of locally acting nasal spray suspension products. In: Respiratory Drug Delivery Europe 2015. Dalby RN, Byron PR, Peart J et al. (Eds). DHI Publisher, IL, USA, 121-130 (2015).

- Reviews the reasons why the low cost and speed of in vitro evaluation with nasal cast models makes them tempting for evaluation of nasal delivery performance.

5. Baraniuk JN. Neural regulation of mucosal function. Pulm. Pharmacol. Ther. 21(3), 442-448 (2008).

6. Djupesland PG, Skretting A. Nasal deposition and clearance in man: comparison of a bidirectional powder device and a traditional liquid spray pump. J. Aerosol Med. Pulm. Drug Deliv. 25(5), 280-289 (2012).

-• Nasal deposition study using the Optinose cast comparing deposition using EDS-FLU (XHANCE ${ }^{\circledR}$ ) with deposition using a conventional intranasal spry (Nasonex ${ }^{\circledR}$ ).

7. Cole P. Nasal respiratory function. In: The Nose. Mosby-Year Book Inc, MO, USA, 3-60 (1993). 
8. Suman JD, Laube BL, Dalby R. Validity of in vitro tests on aqueous spray pumps as surrogates for nasal deposition, absorption and biologic response. J. Aerosol. Med. 19(4), 510-521 (2006).

9. US Food and Drug Administration Center for Drug Evaluation and Research. Draft guidance for industry: bioavailability and bioequivalence studies for nasal aerosols and nasal sprays. www.fda.gov/downloads/Drugs/.../Guidances/ucm070111.pdf

10. Xi J, Yuan JE, Zhang Y et al. Visualization and quantification of nasal and olfactory deposition in a sectional adult nasal airway cast. Pharm. Res. 33(6), 1527-1541 (2016).

11. Koken Co., Ltd. www.kokenmpc.co.jp/english/products/life_simulation_models/medical_education/lm-005/.

12. Palmer JN, Jacobson KW, Messina JC, Kosik-Gonzalez C, Djupesland PG, Mahmoud RA. EXHANCE-12: 1-year study of the exhalation delivery system with fluticasone (EDS-FLU) in chronic rhinosinusitis. Int. Forum Allergy Rhinol. 8(8), 869-876 (2018).

13. Guo Y, Laube B, Dalby R. The effect of formulation variables and breathing patterns on the site of nasal deposition in an anatomically correct model. Pharm. Res. 22(11), 1871-1878 (2005).

- Early deposition study of conventional intranasal spray using the Koken cast.

14. Kundoor V, Dalby RN. Assessment of nasal spray deposition pattern in a silicone human nose model using a color-based method. Pharm. Res. 27(1), 30-36 (2010).

15. Kundoor V, Dalby RN. Effect of formulation- and administration-related variables on deposition pattern of nasal spray pumps evaluated using a nasal cast. Pharm. Res. 28(8), 1895-1904 (2011).

16. Pu Y, Goodey AP, Fang X, Jacob K. A comparison of the deposition patterns of different nasal spray formulations using a nasal cast. Aerosol Sci. Technol. 48(9), 930-938 (2014).

17. Castile J, Cheng YH, Simmons B et al. Development of in vitro models to demonstrate the ability of PecSys ${ }^{\circledR}$, an in situ nasal gelling technology, to reduce nasal run-off and drip. Drug Dev. Ind. Pharm. 39(5), 816-824 (2013).

18. Djupesland PG, Messina J, Mahmoud R. A critical evaluation of the validity of casts for in vitro evaluation of locally acting, nasally administered drug products. Presented at: COSM 2017, CA, USA, 26-30 April 2017.

19. Liu Y, Johnson MR, Matida EA et al. Creation of a standardized geometry of the human nasal cavity. J. Appl. Physiol. (1985). 106(3), 784-795 (2009).

-. Describes the creation of a standardized geometry of the human nasal cavity using computed tomography scans of nasal airways of healthy subjects. These standards were used as 'normative' mean values for comparison in our study.

20. Djupesland PG. Nasal drug delivery devices: characteristics and performance in a clinical perspective - a review. Drug Deliv. Transl. Res. 3(1), 42-62 (2013).

- A review of the nasal anatomy and physiology characteristics that influence intranasal drug delivery, nasal drug-delivery devices and the clinical aspects of nasal deposition and clearance. The lack of validation of any cast dimensions and the inability of casts to reproduce important dynamic aspects of nasal anatomy and physiology are discussed.

21. Fodil R, Brugel-Ribere L, Croce $\mathrm{C}$ et al. Inspiratory flow in the nose: a model coupling flow and vasoerectile tissue distensibility. J. Appl. Physiol. (1985). 98(1), 288-295 (2005).

22. Djupesland PG, Rotnes JS. Accuracy of acoustic rhinometry. Rhinology. 39(1), 23-27 (2001).

23. Hilberg O. Objective measurement of nasal airway dimensions using acoustic rhinometry: methodological and clinical aspects. Allergy. 57(Suppl. 70), 5-39 (2002).

24. Guilmette RA, Cheng YS, Griffith WC. Characterising the variability in adult human nasal airway dimensions. Ann. Occup. Hyg. 41(Suppl. 1), 491-496 (1997).

25. Gomes Ade O, Sampaio-Teixeira AC, Trindade SH, Trindade IE. Nasal cavity geometry of healthy adults assessed using acoustic rhinometry. Braz. J. Otorhinolaryngol. 74(5), 746-754 (2008).

- Study setting the reference values for nasal cavity cross-section geometry in healthy adults using acoustic rhinometry.

26. Zhao K, Jiang J. What is normal nasal airflow? A computational study of 22 healthy adults. Int. Forum Allergy Rhinol. 4(6), 435-446 (2014).

27. Leach CL, Chand R, Kuehl PJ. Human clinical nasal deposition and retention from nonaqueous and aqueous steroid inhalers. In: Respiratory Drug Delivery 2016. Dalby RN, Byron PR, Peart J et al. (Eds). Virginia Commonwealth University, Richmond, VA, pp193-202 (2016).

-. This in vivo gamma study demonstrates that the anterior 'hot-spot', which is due to the highly concentrated deposition in the narrow nasal valve region, is typical of delivery with conventional nasal spray devices.

28. Emanuel IA, Blaiss MS, Meltzer EO, Evans P, Connor A. Nasal deposition of ciclesonide nasal aerosol and mometasone aqueous nasal spray in allergic rhinitis patients. Am. J. Rhinol. Allergy. 28(2), 117-121 (2014). 